\title{
Evaluación de cuatro bioestimulantes y tres sustratos en la propagación de Vallea stipularis L.f.
}

\section{(Evaluation of four bioestimulants and three sustrates in the propagation of Vallea stipularis L.f.)}

\author{
Sonia Rosero ${ }^{1}$, Norma Erazo ${ }^{1}$, Miguel Guallpa ${ }^{1}$, Marlene Usigña $^{1}$
}

\begin{abstract}
Resumen:
La investigación consistió en evaluar cuatro bioestimulantes y tres sustratos para la propagación de Vallea stipularis L.f., en condiciones de invernadero. Los bioestimulantes, correspondieron a: enraizador hormonas vegetales (B1), agrohormonas 10-10-10 (B2), bioestimulante ESPOCH (B3) y Gron Gibb (B4). Los sustratos, consistieron en: $50 \%$ tierra negra $+25 \%$ turba $+25 \%$ arena (S1); $50 \%$ de tierra negra $+25 \%$ turba $+25 \%$ humus (S2) y $50 \%$ turba $+50 \%$ humus (S3). Para el estudio, se utilizó diseño completo al azar (DCA) con arreglo factorial, se determinó el coeficiente de variación y la prueba de Tukey al 5\% para la separación de medias. La aplicación de los bioestimulantes en los sustratos se realizó al momento de la siembra. De acuerdo con los resultados obtenidos, los mejores bioestimulantes correspondieron a B1 (enraizador hormonas vegetales), aplicado en las semillas por 15 minutos, con lo cual se obtuvo el mayor porcentaje de emergencia $(70,96 \%)$; con el bioestimulante B2 (agrohormonas 10-10-10), aplicado por cinco minutos se obtuvo $70.67 \%$ y con B3 (Gron Gibb) 70,37\%. El mejor sustrato para la emergencia de plantas, fue: turba $50 \%+$ humus $50 \%$ ) con $73.67 \%$ de emergencia, donde se logró la mayor altura plantas y longitud de raíz, no así, el bioestimulante ESPOCH (microoganismos), reportó un $67,11 \%$ de emergencia.
\end{abstract}

Palabras clave: bioestimulantes; emergencia; propagación; sustratos; Vallea stipularis.

\begin{abstract}
:
The research consisted in evaluating four biostimulants and three substrates for the propagation of Vallea stipularis L.f., in greenhouse conditions. The biostimulants corresponded to: plant hormone rooting (B1), agrohormones 10-10-10 (B2), ESPOCH biostimulant (B3) and Gron Gibb (B4). The substrates consisted of: $50 \%$ black soil $+25 \%$ peat $+25 \%$ sand (S1); $50 \%$ black soil $+25 \%$ peat $+25 \%$ humus (S2) and $50 \%$ peat $+50 \%$ humus (S3). For the study, it was used a randomized complete design (RCD) with factorial arrangement, it was determined the coefficient of variation and the Tukey test at $5 \%$ for the separation of means. The application of the biostimulants in the substrates was done at the time of planting. According to the results obtained, the best biostimulants corresponded to B1 (plant hormone rooting), applied in the seeds for 15 minutes, with which the highest emergency percentage was obtained $(70.96 \%)$; with biostimulant B2 (agrohormones 10-10-10), applied for five minutes it was obtained $70.67 \%$ and with B3 (Gron Gibb) $70.37 \%$. The best substrate for emergence of plants was: $50 \%$ peat + $50 \%$ humus) with $73.67 \%$ of emergence, where the highest plant height and root length were achieved, instead, the ESPOCH biostimulant (microoganisms) reported a $67,11 \%$ emergency.
\end{abstract}

Keywords: bio stimulants; emergency; propagation; substrates; Vallea stipularis.

\footnotetext{
${ }^{1}$ Escuela Superior Politécnica de Chimborazo, Riobamba - Ecuador ( \{s_rosero, norma.erazo, miguel.guallpa,m_usigña \} @espoch.edu.ec )
} 


\section{Introducción}

Una de las preocupaciones del sector forestal, es la propagación de especies, sobre todo de especies nativas, de las cuales se tiene poca información, como el sacha capulí (Vallea stipularis). El sacha capulí, debido a su importancia para los ecosistemas altoandinos del Ecuador y sus pobladores, merece repoblarlo, mediante el uso de productos que activen los procesos fisiológicos. Se menciona a los bioestimulantes agrícolas, los cuales actúan sobre la fisiología vegetal para mejorar el enraizamiento y prendimiento de estructuras de propagación.

De acuerdo con el inventario botánico de las especies forestales del bosque protector de la microcuenca del Río Blanco, se determinó la existencia de especies vegetales con altos potenciales económicos y ecológicos, que están desapareciendo considerablemente, este es el caso de V. stipularis L.f. con el 1,5\% de presencia (Conchago, 2008). Esta especie, tiene importancia para forestación, agroforestería, restauración de paisajes, protección de suelos y aprovechamiento maderable y no maderable (Prado \& Valdebenito, 2000).

Actualmente, constituye una especie de aprovechamiento condicionado en el manejo sustentable del Bosque Andino, según la Norma Técnica № 128 (Ministerio del Ambiente, 2011). Ante esta realidad, se planteó realizar la presente investigación para mejorar su propagación.

La propagación del sacha capulí generalmente se realiza mediante semillas sin ningún tratamiento pregerminativo, por lo que se obtienen bajos porcentajes de emergencia en el campo, razón por la cual, los programas de restauración ecológica y/o reforestación consideran indispensable mejorar los métodos de tratamientos de semillas, a fin de incrementar el número de individuos para satisfacer la demanda local, dada la utilidad de esta especie para la conservación de las zonas restauradas y su uso (Vázquez \& Batis, 1996).

Otro aspecto importante que tiene relación con la emergencia de las semillas, constituye el tipo de sustrato, el mismo que se encuentra fuertemente influido por las características físico-químicas, ya que puede favorecer o entorpecer el proceso (Niembro \& Fierros, 1990). Los sustratos en la emergencia de semillas de las especies forestales ha tenido una atención especial (Vega, 1986).

El éxito de la propagación de una especie, depende de las condiciones fisiológicas de la planta semillera, la concentración de los reguladores de crecimiento, las condiciones meteorológicas, el tipo de propágulo, los plaguicidas y la influencia del sustrato (Aloisio et al., 2003). Por tanto, en este estudio, se planteó, evaluar el efecto de bioestimulantes y sustratos en la propagación sexual del V. stipularis L.f. 


\section{Materiales y métodos}

\section{1 Área de estudio}

La presente investigación se realizó en el Vivero Forestal del Consorcio Río Blanco, que funciona en la Granja Agrícola del Ministerio de Agricultura, Ganadería, Acuacultura y Pesca (MAGAP), de la parroquia Quimiag, ubicada al noreste del cantón Riobamba, provincia de Chimborazo, a 16 $\mathrm{km}$ de la cabecera cantonal. Geográficamente el vivero se localiza en el punto de referencia: UTM Zona 17S Datum WGS $84 X=770667 ; Y=9816584$. Se encuentra ubicada a una altitud de 2752 msnm. La precipitación anual es de $500 \mathrm{~mm}$, la temperatura media está entre 11 a $15^{\circ} \mathrm{C}$ y hay una humedad relativa media de 68-75 \%. Datos: serie 1984-2014 de la Estación Meteorológica de la ESPOCH (2014). Se categoriza dentro de la formación: Matorral Húmedo Montano de los Andes del Norte y Centro (MAE, 2012). El rango de temperatura del invernadero registrada durante el período del ensayo fue de: 18 - 35ํ․

\subsection{Lugar de recolección de semilla}

En la Comunidad "El Toldo", sector "La Calera", a una altitud 3174 msnm, se recolectaron las semillas en un remanente de bosque de la zona directamente de cada árbol entre el 20 al 25 de julio de 2012, los frutos se colocaron bajo sombra para el secado; se procedió a seleccionar las semillas con buenas características en sanidad, color, forma y tamaño (Jará \& Ordóñez, 1999) (Ballesteros et al., 2015). Se aplicó una prueba de flotación para descartar las semillas no viables (Mulawarman et al., 2003).

\subsection{Preparación de los sustratos y siembra}

El semillero se elaboró bajo condiciones de invernadero, de 9 metros de longitud por $1 \mathrm{~m}$ de ancho sobre nivel con $10 \mathrm{~cm}$ de altura. Área dividida en 39 parcelas de 23 por $100 \mathrm{~cm}$.

La preparación de los sustratos, se inició con el tamizado de cada componente (tierra negra, turba, arena, humus y tierra agrícola), luego se establecieron las mezclas de los tres sustratos (Tabla1), la desinfección con Pentacloronitrobenzeno (P.C.N.B) en una dosis de $100 \mathrm{~g} / \mathrm{m}^{3}$.

La siembra se efectuó en surcos, a $1 \mathrm{~cm}$ de profundidad, $8 \mathrm{~cm}$ de distancia entre surco y $2 \mathrm{~cm}$ entre semillas, colocando 50 semillas por surco y 150 semillas por parcela neta, fueron cubiertas con una fina capa del mismo sustrato.

Se estableció un diseño Completamente al Azar (DCA) en arreglo combinatorio, con 3 repeticiones. Los tratamientos se observan en la Tabla 1.

\subsection{Características de los bioestimulantes}

En la Tabla 2, se muestra la composición química y concentración de ciertos elementos que 
forman parte de los bioestimulantes.

Tabla 1. Tratamientos en estudio para la propagación sexual

\begin{tabular}{|c|c|l|}
\hline No Trat & Código & \multicolumn{1}{c|}{ Descripción } \\
\hline T1 & B1S1 & Enraizador $\mathrm{H}-\mathrm{V} 3 \mathrm{cc} / \mathrm{L}$ agua $\times 15 \mathrm{~min} .+50 \%$ tierra negra $+25 \%$ turba+25\% arena \\
\hline T2 & B1S2 & Enraizador $\mathrm{H}-\mathrm{V} 3 \mathrm{cc} / \mathrm{L}$ agua $\times 15 \mathrm{~min} .+50 \%$ tierra negra $+25 \%$ turba $+25 \%$ humus \\
\hline T3 & B1S3 & Enraizador $\mathrm{H}-\mathrm{V} 3 \mathrm{cc} / \mathrm{L}$ agua $\times 15 \mathrm{~min} .+50 \%$ turba $+50 \%$ humus \\
\hline T4 & B2S1 & Agrohormonas $10 \mathrm{cc} / \mathrm{L}$ agua $\times 5 \mathrm{~min} .+50 \%$ tierra negra $+25 \%$ turba $+25 \%$ arena \\
\hline T5 & B2S2 & Agrohormonas $10 \mathrm{cc} / \mathrm{L}$ agua $\times 5 \mathrm{~min} .+50 \%$ tierra negra $+25 \%$ turba $+25 \%$ humus \\
\hline T6 & B2S3 & Agrohormonas $10 \mathrm{cc} / \mathrm{L}$ agua $\times 5 \mathrm{~min} .+50 \%$ turba $+50 \%$ humus \\
\hline T7 & B3S1 & Bioest. ESPOCH solución directa $\times 30$ min. $+50 \%$ tierra negra $+25 \%$ turba $+25 \%$ arena \\
\hline T8 & B3S2 & Bioest. ESPOCH solución directa $\times 30$ min. $+50 \%$ tierra negra $+25 \%$ turba $+25 \%$ arena \\
\hline T9 & B3S3 & Bioest. ESPOCH solución directa $\times 30$ min. $+50 \%$ tierra negra $+25 \%$ turba $+25 \%$ arena \\
\hline T10 & B4S1 & Gron Gibb $5 \mathrm{~g} / \mathrm{L}$ agua $\times 5$ min. $+50 \%$ tierra negra $+25 \%$ turba $+25 \%$ arena \\
\hline T11 & B4S2 & Gron Gibb $5 \mathrm{~g} / \mathrm{L}$ agua $\times 5$ min. $+50 \%$ tierra negra $+25 \%$ turba $+25 \%$ humus \\
\hline T12 & B4S3 & Gron Gibb $5 \mathrm{~g} / \mathrm{L}$ agua $\times 5$ min. $+50 \%$ turba $+50 \%$ humus \\
\hline T13 & Testigo & S/T + Tierra agrícola \\
\hline
\end{tabular}

Bioestimulantes: Enraizador hormonas vegetales (B1), Agrohormonas 10-10-10 (B2), Bioestimulante ESPOCH (B3), Gron Gibb (B4). Sustratos: $50 \%$ tierra negra $+25 \%$ turba $+25 \%$ arena (S1), $50 \%$ tierra negra $+25 \%$ turba $+25 \%$ humus (S2) y $50 \%$ turba $+50 \%$ humus (S3).

Tabla 2. Composición química y concentración de algunos elementos de los bioestimulantes

\begin{tabular}{|c|c|c|c|}
\hline \multicolumn{4}{|c|}{ 1. Enraizador Hormonas Vegetales } \\
\hline Producto & Concentración & Producto & Concentración \\
\hline Ácido indobutírico (IBA) & $0.05 \%$ & Otras Auxinas & $0.005 \%$ \\
\hline Ácido naftilacético (ANA) & $0.09 \%$ & Fósforo asimilable & $1 \%$ \\
\hline Ácido giberélico & $0.01 \%$ & Nitrógeno orgánico & $0.5 \%$ \\
\hline Citoquininas & $0.01 \%$ & Hidratos de Carbono & $2.5 \%$ \\
\hline \multicolumn{4}{|c|}{ 2. Agrohormonas - concentrado $10-10-10$} \\
\hline Producto & Concentración & Producto & Concentración \\
\hline Nitrógeno & $10 \%$ & Aminoácidos & Concentrados \\
\hline Fósforo & $10 \%$ & Ácidos Húmicos & Concentrados \\
\hline Potasio & $10 \%$ & Ácidos Orgánicos & $2.5 \%$ \\
\hline Magnesio & $4 \%$ & $\begin{array}{c}\text { Giberelinas, Citoquininas, } \\
\text { Auxinas }\end{array}$ & $0.08 \%$ \\
\hline Azufre & $4 \%$ & Proteínas, Enzimas & Abundantes \\
\hline Boro & $4 \%$ & Vitaminas B1, B6, B12 & Concentrados \\
\hline $\mathrm{Mn}, \mathrm{Fe}, \mathrm{Zn}, \mathrm{Cu}, \mathrm{Mo}, \mathrm{Ni}, \mathrm{Co}$ & $2 \%$ & Regulador de $\mathrm{pH}$ & $5-6$ \\
\hline \multicolumn{4}{|c|}{ 3. Gron Gibb. $10 \%$} \\
\hline Ingrediente activo & Porcentaje & & \\
\hline Giberelinas A3 & $10 \%$ & & \\
\hline Ingredientes inertes & $90 \%$ & & \\
\hline
\end{tabular}

Fuente: ${ }^{1,2}$ Agro-Orgánica ( 2010), ${ }^{3}$ Vademécum Agrícola (2008).

\section{Estimulador Biológico - ESPOCH}

Este producto de origen biológico, es el resultado de la descomposición anaeróbica de residuos vegetales procedente de un bosque andino nativo, enriquecido con salvado de trigo y melaza; 
multiplicados en medio enriquecido por 3 meses, bajo condiciones de invernadero (temperatura media: $25^{\circ} \mathrm{C}$ ) en la Facultad de Recursos Forestales de la ESPOCH (Erazo, 2009).

\subsection{Contenido nutricional de los sustratos}

El reporte del análisis químico de los sustratos se indica en la Tabla 3.

Tabla 3. Análisis químico de los sustratos en estudio

\begin{tabular}{|c|c|c|c|c|c|c|c|}
\hline \multirow{2}{*}{ Sustratos } & MO & pH & \multicolumn{5}{|c|}{ \% Elementos } \\
\cline { 2 - 8 } & $\%$ & & $\mathrm{~N}$ & $\mathrm{P}$ & $\mathrm{K}$ & $\mathrm{Ca}$ & $\mathrm{Mg}$ \\
\hline Testigo & $4,2 \mathrm{M}$ & 7,7 L.Alc & 0,34 & 0,34 & 0,13 & 0,46 & 0,03 \\
\hline S1 & $8,8 \mathrm{~A}$ & 5,9 L.Alc. & 0,39 & 0,34 & 0,10 & 0,40 & 0,02 \\
\hline S2 & $10,0 \mathrm{~A}$ & 5,9 L. Ac & 0,78 & 0,34 & 0,10 & 0,32 & 0,09 \\
\hline S3 & $14,0 \mathrm{~A}$ & 5,8 L. Ac & 1,04 & 0,34 & 0,09 & 0,34 & 0,03 \\
\hline
\end{tabular}

Materia orgánica (MO), Potencial de hidrógeno $(\mathrm{pH})$, Nitrógeno $(\mathrm{N})$, Fósforo $(\mathrm{P})$, Potasio $(\mathrm{K})$, Calcio (Ca), Magnesio (Mg), Ligeramente alcalino (L.Acl.), Ligeramente ácido (L.Ac.), Alto (A) y Medio (M).

Fuente: Laboratorio de suelos de la Facultad de Recursos Naturales de la ESPOCH (2012).

\subsection{Labores culturales}

El riego en el semillero, se realizó en la mañana y tarde a partir del monitoreo periódico de la humedad con el tensiómetro en los sustratos, considerando un rango de 50 a 70 \%; durante las primeras cuatro semanas, con una bomba de mochila y posteriormente con el sistema de micro aspersión, a partir de la emergencia, el riego se dio cada dos días.

En base a la observación de síntomas de enfermedades y plagas en las plantas, se tomaron muestras de las plantas y sustratos para su análisis en laboratorio de Fitopatología de la FRN$\mathrm{ESPOCH}$, el reporte fue de damping off; $y$ los insectos de la familia Cucurlionidae defoliadores de hojas.

El control químico fitosanitario se efectuó una vez receptado los resultados de laboratorio de acuerdo con un período de aplicación de 8 días para el control de hongos y de 15 días para insectos, babosas conjuntamente con la regulación del riego y la temperatura (Tabla 4).

La actividad de deshierba se realizó a los 8 días después de la siembra en los tratamientos. Luego se realizó esta actividad con intervalos de 15 días durante el período de investigación (Guevara \& Villacrés, 1999).

\subsection{Evaluación en el semillero}

Se evaluó el porcentaje de emergencia a los 30, 60, 90 y 120 días. Para el porcentaje de emergencia (PE) se aplicó la ecuación (1).

La medición de altura de planta (AP) se realizó desde los 60 días con intervalos de 30 días hasta 
los 120 días y la longitud de raíces (LR) a los 30 y 120 días.

$$
P E=\frac{N^{\circ} \text { de semillas emergidas } \times 100 \%}{N^{\circ} \text { total de semillas } x \text { tratamiento }}
$$

Tabla 4. Control químico fitosanitario durante el ensayo

\begin{tabular}{|c|c|c|c|}
\hline $\begin{array}{c}\text { Plagas y } \\
\text { enfermedades }\end{array}$ & Producto & Dosis & $\begin{array}{l}\text { Frecuencia de aplicación } \\
\text { (días) }\end{array}$ \\
\hline Desinfectante de suelo & $\begin{array}{l}\text { Pentacloronitrobenzeno } \\
\text { (P.C.N.B) }\end{array}$ & $100 \mathrm{~g} \mathrm{~m}^{3}-^{1}$ & Una sola aplicación \\
\hline \multirow[t]{3}{*}{ Damping off } & Metalaxyl-M+Cloratonil & $\begin{array}{rll}5 & \mathrm{~g} & \mathrm{~L}- \\
\text { 1agua } & & \end{array}$ & 8 \\
\hline & Propamocarb & $\begin{array}{l}1 \mathrm{~cm}^{3} \quad \mathrm{~L}- \\
\text { 1agua }\end{array}$ & 8 \\
\hline & Novak 70 PM & $\begin{array}{r}5 \mathrm{~g} \quad \mathrm{~L} \\
\text { 1agua }\end{array}$ & 8 \\
\hline Lancha & Cimoxanil + Mancozeb & $\begin{array}{r}5 \mathrm{~g} \quad \mathrm{~L} \\
\text { 1agua }\end{array}$ & 15 \\
\hline Insectos, babosas & Profenofos (Curacron) & $\begin{array}{l}1 \mathrm{~cm}^{3} \quad \mathrm{~L} \quad- \\
\text { 1agua }\end{array}$ & 15 \\
\hline Insectos, babosas & Chlorpyrifos & $\begin{array}{l}1 \mathrm{~cm}^{3} \mathrm{~L} \quad- \\
\text { 1agua }\end{array}$ & 15 \\
\hline Regulador de $\mathrm{pH}$ & Indicate-5 & $\begin{array}{l}2 \mathrm{~cm}^{3} \quad \mathrm{~L} \quad- \\
\text { 1agua }\end{array}$ & En cada aplicación \\
\hline Fijador & Break Thru & $\begin{array}{l}0,1 \mathrm{~cm}^{3} \quad \mathrm{~L} \quad- \\
\text { 1agua }\end{array}$ & En cada aplicación \\
\hline
\end{tabular}

\subsection{Análisis estadístico}

Para las variables, porcentaje de emergencia (PE), altura de planta (AP) y longitud de raíces (LR) se aplicó un análisis de varianza, prueba de Tukey $(\alpha=0,05)$, utilizando el programa INFOSTAT (Balzarini et al., 2008).

\section{Resultados}

\subsection{Porcentaje de emergencia}

El análisis de varianza para la variable emergencia de plantas a los 30, 60, 90 y 120 días después de la siembra, demostró ser altamente significativos al 95\% de probabilidad estadística, referente a los factores: bioestimulantes, sustratos y la interacción factores versus testigo respectivamente (p-valor, Tabla 5).

Para la variable porcentaje de emergencia (Tabla 6), se muestra de manera independiente, el efecto altamente significativo para los factores: bioestimulantes, sustratos y la interacción testigo 
versus el resto. De acuerdo con ello, la prueba de rango múltiple de Tukey a los 30 días, el primer lugar lo ocupa el bioestimulante B4 (Semillas + Gron Gibb x 5 minutos) que presentó una media de 2.44\%; a los 60, 90, y 120 días se ubicó como el más alto B1 (Semillas+Enraizador de Hormonas Vegetales x 15 minutos), con una media de 24.15, 69.7 y $70.96 \%$ (a) respectivamente. En contraste el valor más bajo correspondió al efecto de B3 (Semillas+ Bioestimulante ESPOCH x 30 minutos) con promedios de 1.48, 20.3, 65.56 y $67.11 \%$ (b).

Tabla 5. Análisis de varianza de la variable emergencia.

\begin{tabular}{|c|c|c|c|c|c|c|}
\hline Variable medida & F.V & GL & sc & CM & Fc & p-valor \\
\hline \multirow{7}{*}{ Emergencia 30 días } & Bioestimulantes & 3 & 4,87 & 1,62 & 5,0625 & 0,007 \\
\hline & Sustratos & 2 & 48,22 & 24,11 & 75,344 & 0,000 \\
\hline & Interacción BxS & 6 & 3,81 & 0,64 & 2 & 0,102 \\
\hline & Factores versus testigo & 1 & 11,7 & 11,7 & 36,51 & 0,0001 \\
\hline & Error & 26 & 8,33 & 0,32 & & \\
\hline & CV \% & & & 29,84 & & \\
\hline & Media & & & 1,90 & & \\
\hline \multirow{7}{*}{ Emergencia 60 días } & Factor B & 3 & 85,57 & 28,52 & 9,70 & 0,0002 \\
\hline & Factor S & 2 & 462,71 & 231,36 & 78,69 & 0,0000 \\
\hline & Interacción BxS & 6 & 22,98 & 3,83 & 1,30 & 0,2909 \\
\hline & Factores versus testigo & 1 & 116,9 & 116,9 & 39,73 & 0,0001 \\
\hline & Error & 26 & 76,50 & 2,94 & & \\
\hline & $\mathrm{CV} \%$ & & & 7,64 & & \\
\hline & Media & & & 22,44 & & \\
\hline \multirow{7}{*}{$\begin{array}{l}\text { Emergencia } 90 \\
\text { días }\end{array}$} & Factor B & 3 & 107,33 & 35,78 & 11,50 & 0,0001 \\
\hline & Factor S & 2 & 535,16 & 267,58 & 86,04 & 0,0000 \\
\hline & Interacción BxS & 6 & 24,84 & 4,14 & 1,33 & 0,2791 \\
\hline & Factores versus testigo & 1 & 819,65 & 819,65 & 263,41 & 0,0001 \\
\hline & Error & 26 & 80,90 & 3,11 & & \\
\hline & $\mathrm{CV} \%$ & & & 2,62 & & \\
\hline & Media & & & 67,21 & & \\
\hline \multirow{7}{*}{$\begin{array}{c}\text { Emergencia120 } \\
\text { días }\end{array}$} & Factor B & 3 & 86,94 & 28,98 & 11,36 & 0,0001 \\
\hline & Factor S & 2 & 368,38 & 184,19 & 72,23 & 0,0000 \\
\hline & Interacción BxS & 6 & 34,95 & 5,82 & 2,28 & 0,0667 \\
\hline & Factores versus testigo & 1 & 942,03 & 942,03 & 369,05 & 0,0001 \\
\hline & Error & 26 & 66.37 & 2,55 & & \\
\hline & CV \% & & & 2,34 & & \\
\hline & Media & & & 68,36 & & \\
\hline
\end{tabular}

Factor B (Bioestimulantes), Factor S (Sustratos).

La prueba de Tukey indica que el factor sustratos registró valores altamente significativos a los 30,60, 90 y 120 días, en donde el sustrato 3 compuesto por Turba 50\%+Humus 50\%, ocupó el rango (a) con promedios de 3,5, 27.89, 73.22, 73.67\%; mientras que el sustrato 1 compuesto por Tierra negra $50 \%+$ Turba $25 \%+$ Arena $25 \%$ presentó los valores más bajos en los 4 períodos de 
evaluación previstos (b). La interacción testigo vs resto, resultaron altamente significativos todos los factores en relación al testigo.

Tabla 6. Efecto de factores y el contraste en el porcentaje de emergencia a los 30, 60, 90 y 120 días después de la siembra.

\begin{tabular}{|l|c|c|c|c|}
\hline Tratamientos & \multicolumn{4}{|c|}{ Porcentaje de emergencia } \\
\hline Bioestimulantes & 30 días & $\mathbf{6 0}$ días & $\mathbf{9 0}$ días & $\mathbf{1 2 0}$ días \\
\hline Enraizador Hormonas Vegetales & $2,0 \mathrm{ab}$ & $24,15 \mathrm{a}$ & $69,7 \mathrm{a}$ & $70,96 \mathrm{a}$ \\
\hline Agro Hormonas & $2,3 \mathrm{ab}$ & $23,63 \mathrm{a}$ & $69,56 \mathrm{a}$ & $70,67 \mathrm{a}$ \\
\hline Bioestimulante ESPOCH & $1,48 \mathrm{~b}$ & $20,3 \mathrm{~b}$ & $65,56 \mathrm{~b}$ & $67,11 \mathrm{~b}$ \\
\hline Gron Gibb & $2,44 \mathrm{a}$ & $23,7 \mathrm{a}$ & $69,33 \mathrm{a}$ & $70,37 \mathrm{a}$ \\
\hline Sustratos & & & & \\
\hline TN 50\%+T 25\%+A 25\% & $0,67 \mathrm{~b}$ & $19,5 \mathrm{c}$ & $63,78 \mathrm{c}$ & $65,83 \mathrm{c}$ \\
\hline TN 50\%+T 25\%+H 25\% & $2,0 \mathrm{ab}$ & $21,44 \mathrm{~b}$ & $68,61 \mathrm{~b}$ & $69,83 \mathrm{~b}$ \\
\hline T 50\%+H 50\% & $3,5 \mathrm{a}$ & $27,89 \mathrm{a}$ & $73,22 \mathrm{a}$ & $73,67 \mathrm{a}$ \\
\hline Testigo & $0 \mathrm{~b}$ & $16,45 \mathrm{~b}$ & $51,33 \mathrm{~b}$ & $51,33 \mathrm{~b}$ \\
\hline Resto & $2,06 \mathrm{a}$ & $22,94 \mathrm{a}$ & $68,54 \mathrm{a}$ & $69,78 \mathrm{a}$ \\
\hline
\end{tabular}

Valores seguidos por la misma letra, en la misma columna, no son estadísticamente diferentes (Tukey 0,05).

\subsection{Altura de planta}

El análisis de varianza para la variable altura de planta a los 60 días, mostró que el factor bioestimulantes es significativo al $95 \%$ de probabilidad estadística ( $p<0,0295$, Tabla 7 ). En cambio, a los 90 días (Tabla 7), registró su efecto estadístico altamente significativo el factor sustratos al $99 \%$ de probabilidad $(p<0,0011)$ y los factores versus testigo $(p<0,0001)$. Para los 120 días se evidencia un comportamiento similar como el alcanzado a los 90 días del factor sustratos $(p<0,0002)$ y de los factores versus testigo $(p<0,0001)$ cuyo efecto estadístico es altamente significativo.

Según la prueba de rango múltiple de Tukey para el factor bioestimulantes (Tabla 8), determinó la mayor altura de plantas a los 60 días, se obtuvo con B4 (Semillas + Gron Gibb x 5 minutos) con una media de $6.38 \mathrm{~cm}$ (a); no mostró valor significativo el factor bioestimulantes a los 90 y 120 días respectivamente. El valor más bajo ocupó el B3 (Semillas+ Bioestimulante ESPOCH x 30 minutos) (b) con una media de $6.17 \mathrm{~cm}(\mathbf{b})$.

\subsection{Longitud de raíces}

El análisis de varianza para la variable longitud de raíces a los 30 días, mostró un efecto estadístico no significativo de los factores: bioestimulantes, sustratos, interacción y los factores versus testigo (Tabla 9).

A los 120 días el análisis de varianza para la longitud de raíces, demostró su efecto altamente significativo al 99\% de probabilidad estadística para los factores: bioestimulantes $(p<0,0001)$, sustratos $(p<0,0000)$ y la interacción factores versus testigo $(p<0,000$, Tabla9). De acuerdo con el 
factor bioestimulante (Tabla 10), la prueba de rango múltiple de Tukey determinó la mayor longitud de raíces a los 120 días, se reportó a B2 (Semillas + Agrohormonas x 5 minutos) con un promedio de $6.20 \mathrm{~cm}(\mathbf{a})$; en contraste, el B3 (Bioestimulante ESPOCH x 30 minutos) con $5.68 \mathrm{~cm}$ (b)

Tabla 7. Análisis de varianza de la variable altura de planta a los 60, 90, y 120 días.

\begin{tabular}{|c|c|c|c|c|c|c|}
\hline Variable medida & F.V & GL & SC & CM & Fc & p-valor \\
\hline \multirow{7}{*}{$\begin{array}{l}\text { Altura de planta } \\
\quad 60 \text { días }\end{array}$} & Factor B & 3 & 0,20 & 0,07 & 3,50 & 0,0295 \\
\hline & Factor S & 2 & 0,01 & 0,0004 & 0,02 & 0,9792 \\
\hline & Interacción BxS & 6 & 0,04 & 0,01 & 0,50 & 0,8025 \\
\hline & Factores versus testigo & 1 & 0,02 & 0,02 & 1,62 & 0,2138 \\
\hline & Error & 26 & 0,39 & 0,02 & & \\
\hline & CV \% & & & 1,95 & & \\
\hline & Media & & & 6,28 & & \\
\hline \multirow{7}{*}{$\begin{array}{l}\text { Altura de planta } \\
90 \text { días }\end{array}$} & Factor B & 3 & 0,42 & 0,14 & 1,17 & 0,3414 \\
\hline & Factor S & 2 & 2,15 & 1,07 & 8,92 & 0,0011 \\
\hline & Interacción BxS & 6 & 0.04 & 0,01 & 0,08 & 0,9974 \\
\hline & Factores versus testigo & 1 & 9,8 & 9,8 & 82,57 & 0,0001 \\
\hline & Error & 26 & 3,09 & 0,12 & & \\
\hline & CV \% & & & 3,74 & & \\
\hline & Media & & & 9,22 & & \\
\hline \multirow{7}{*}{$\begin{array}{l}\text { Altura de planta } \\
120 \text { días }\end{array}$} & Factor B & 3 & 1,07 & 0,36 & 1,89 & 0,1553 \\
\hline & Factor S & 2 & 4,65 & 2,33 & 12,26 & 0,0002 \\
\hline & Interacción BxS & 6 & 0,06 & 0,01 & 0,05 & 0,9993 \\
\hline & Factores vs testigo & 1 & 19,38 & 19,38 & 104,03 & 0,0001 \\
\hline & Error & 26 & 4,84 & 0,19 & & \\
\hline & CV \% & & & 4,19 & & \\
\hline & Media & & & 10,30 & & \\
\hline
\end{tabular}

Tabla 8. Efecto de los factores y contraste en la altura de las plantas a los 60,90 y 120 días después de la emergencia $(\mathrm{cm})$

\begin{tabular}{|c|c|c|c|}
\hline Tratamientos & \multicolumn{3}{|c|}{ Altura de planta (cm) } \\
\hline Bioestimulantes & 60 días & 90 días & 120 días \\
\hline Enraizador Hormonas Vegetales & $6,31 a b$ & $9,37 \mathrm{~ns}$ & $10,52 \mathrm{~ns}$ \\
\hline Agro Hormonas & $6,29 a b$ & $9,49 \mathrm{~ns}$ & $10,77 \mathrm{~ns}$ \\
\hline Bioestimulante ESPOCH & $6,17 \mathrm{~b}$ & $9,22 \mathrm{~ns}$ & $10,30 \mathrm{~ns}$ \\
\hline Gron Gibb & $6,38 \mathrm{a}$ & $9,44 \mathrm{~ns}$ & $10,53 \mathrm{~ns}$ \\
\hline \multicolumn{4}{|l|}{ Sustratos } \\
\hline TN 50\% +T 25\%+A 25\% & $6,30 \mathrm{~ns}$ & $9,06 \mathrm{~b}$ & $10,09 \mathrm{c}$ \\
\hline TN 50\%+T 25\%+H 25\% & $6,27 \mathrm{~ns}$ & $9,38 \mathrm{ab}$ & $10,46 \mathrm{~b}$ \\
\hline $\mathrm{T} 50 \%+\mathrm{H} 50 \%$ & $6,29 \mathrm{~ns}$ & $9,65 \mathrm{a}$ & $10,97 \mathrm{a}$ \\
\hline Testigo & $6,19 \mathrm{~ns}$ & $7,48 \mathrm{~b}$ & $7,86 \mathrm{~b}$ \\
\hline Resto & $6,28 \mathrm{~ns}$ & $9,36 \mathrm{a}$ & $10,51 \mathrm{a}$ \\
\hline
\end{tabular}

Valores seguidos por la misma letra, en la misma columna, no son estadísticamente diferentes (Tukey 0,05 ). 
Tabla 9. Análisis de varianza de la variable longitud de raíz a los 30 y 120 días

\begin{tabular}{|c|c|c|c|c|c|c|}
\hline Variable medida & F.V & GL & SC & CM & Fc & p-valor \\
\hline \multirow{7}{*}{$\begin{array}{c}\text { Longitud de raíz a los } 30 \\
\text { días }\end{array}$} & Factor B & 3 & 0,07 & 0,02 & 1,00 & 0,4085 \\
\hline & Factor S & 2 & 0,08 & 0,04 & 2,00 & 0,1556 \\
\hline & Interacción BxS & 6 & 0,01 & 0,0009 & 0,04 & 0,9996 \\
\hline & Factores versus testigo & 1 & 0,04 & 0,04 & 1,67 & 0,2071 \\
\hline & Error & 26 & 0,58 & 0,02 & & \\
\hline & CV \% & & & 28,56 & & \\
\hline & Media & & & 0,52 & & \\
\hline \multirow{7}{*}{$\begin{array}{l}\text { Longitud de raíz a los } \\
120 \text { días }\end{array}$} & Factor B & 3 & 1,27 & 0,42 & 10,50 & 0,0001 \\
\hline & Factor S & 2 & 3,05 & 1,53 & 38,25 & 0,0000 \\
\hline & Interacción BxS & 6 & 0,07 & 0,01 & 0,25 & 0,9549 \\
\hline & Factores versus testigo & 1 & 3,24 & 3,24 & 91,87 & 0,0001 \\
\hline & Error & 26 & 0,92 & 0,04 & & \\
\hline & $\mathrm{CV} \%$ & & & 3,21 & & \\
\hline & Media & & & 5,85 & & \\
\hline
\end{tabular}

Factor B (Bioestimulantes), Factor S (Sustratos).

Tabla 10. Efecto de los factores y contrastes en la longitud de la raíz (cm) a los 120 días después de la siembra.

\begin{tabular}{|l|c|}
\hline Tratamientos & $\begin{array}{c}\text { Longitud de raíz } \\
\text { (cm) }\end{array}$ \\
\hline Bioestimulantes & $\mathbf{1 2 0 ~ d i ́ a s ~}$ \\
\hline Enraizador Hormonas Vegetales & $5,96 \mathrm{ab}$ \\
\hline Agro Hormonas & $6,20 \mathrm{a}$ \\
\hline Bioestimulante ESPOCH & $5,68 \mathrm{~b}$ \\
\hline Gron Gibb & $5,87 \mathrm{ab}$ \\
\hline Sustratos & \\
\hline TN 50\% +T 25\%+A 25\% & $5,53 \mathrm{~b}$ \\
\hline TN 50\%+T 25\%+H 25\% & $6,05 \mathrm{ab}$ \\
\hline T 50\%+H 50\% & $6,21 \mathrm{a}$ \\
\hline Testigo & $4,85 \mathrm{~b}$ \\
\hline Resto & $5,93 \mathrm{a}$ \\
\hline
\end{tabular}

La variable longitud de la raíz a los 120 días (Tabla 10) ocupó el primer lugar (a) con el sustrato 3 (Turba $50 \%$ + Humus 50\%) con una media de $6.21 \mathrm{~cm}$.; y en el último lugar se ubicó el sustrato 1 (Tierra negra $50 \%$ + Turba 25\% + Arena 25\%) con una media de $5.53 \mathrm{~cm}$ (b). La interacción testigo versus resto, resultó altamente significativa para todos los factores en relación con el testigo (Tabla 10).

\section{Discusión}

En general, los factores bioestimulantes y sustratos incrementaron el porcentaje de emergencia, en relación con el testigo de la especie $V$. stipularis L.f., con la aplicación de Gron Gibb y Enraizador Hormonas Vegetales, coincidiendo con lo mencionado según Trujillo, (2010), quien afirma que los bioestimulantes promueven el crecimiento del embrión y la salida de cada plántula, 
es decir, actuaron favorablemente en el proceso de división y elongación de los tejidos, pero debido a las características morfológicas de las semillas deben ser sometidas a algún tipo de tratamientos, ya sea con químicos o bioestimulantes (Añazco, 2000).

Así mismo los sustratos constituyen el medio de soporte para el desarrollo de la plántula. Pues durante la emergencia y el crecimiento radicular proveen de humedad, nutrientes y un adecuado intercambio de aire Fernández (1992). Se atribuye que al realizar la mezcla del 50\% de turba más el $50 \%$ de humus se consiguió un sustrato de buena calidad y con alto contenido de materia orgánica, como lo demuestra el análisis químico del sustrato, que influyó en la emergencia de las plántulas. En este sentido, la siembra de esta especie se lo debe hacer con sustratos que contenga por lo menos el $50 \%$ de materia orgánica ya que esta elevará su temperatura y ayudará a la emergencia de las semillas (Jará \& Ordóñez, 1999).

Con respecto a la influencia del factor bioestimulante, Gron Gibb sobre el crecimiento en altura de la especie en estudio, mostró su acción en los períodos iniciales hasta los 60 días después de la siembra. Pues es conocida una de las aplicaciones prácticas del ácido giberélico para estimular el desarrollo en altura (Trujillo, 2010).

Al igual, que en el proceso de emergencia el sustrato formado por la mezcla del $50 \%$ de turba más el $50 \%$ de humus contribuyeron para alcanzar una mayor altura de planta, el sustrato al estar formado por un buen contenido de materia orgánica, aumentó su drenaje, además elevó sus niveles de $\mathrm{N}, \mathrm{P}, \mathrm{K}$ y $\mathrm{Mg}$, los mismos que mejoran e incrementan el crecimiento de esta variable a partir de los 90 días luego de la siembra de V.stipularis L.f (Añazco, 2000).

La longitud de raíz está influenciada por el sustrato tres al estar formado por tierra negra y humus, existiendo una mayor disponibilidad de materia orgánica y $\mathrm{N}$, facilitando su asimilación, lo cual influye tanto en el crecimiento aéreo y radicular de las plantas (Guevara \& Villacrés, 1999).

\section{Conclusiones y recomendaciones}

El bioestimulante, B1 (Semillas+Enraizador Hormonas Vegetales $\times 15$ minutos) fue el mejor con $70.96 \%$ de emergencia; mientras que para la longitud de la raíz el mejor fue B2 (Semillas +Agro hormonas $x 5$ minutos) con $6.2 \mathrm{~cm}$ de crecimiento a los 120 días.

Se determinó que el sustrato compuesto por Turba 50\% + Humus 50\% (B3) fue el mejor en todas las variables evaluadas, con lo cual se comprobó que la semilla de $V$. stipularis L.f., reaccionó favorablemente a este tipo de sustratos con un alto contenido de materia orgánica y nutrientes según el reporte del análisis físico-químico del laboratorio.

Se sugiere para este tipo de propagación utilizar bioestimulantes a base de hormonas vegetales en una dosis de $3 \mathrm{~cm}^{3} \mathrm{~L}^{-1}$ de agua y un sustrato con el $50 \%$ de Turba $+50 \%$ de Humus, 
debiéndose evaluar sus atributos morfológicos y fisiológicos, que le den la capacidad de adaptarse y desarrollarse en las condiciones edafoclimáticas del sitio definitivo.

\section{Bibliografía}

Agro-Orgánica. (2010). Obtenido de http://www.agrorganica.com

Aloisio, X., Gleison, A., Ivar, W., \& Marcelo Lelis de Oliveira. (2003). Propagação vegetativa de cedro-rosa por miniestaquia. Árvore, 27; 139-143.

Añazco, M. (2000). Producción de plantas. Quito, Ecuador.

Balzarini, M. G., González, L., Tablada, M., Casanoves, F., Di Rienzo, J. A., \& Robledo, C. W. (2008). Infostat. Manual del Usuario. Córdova, Argentina: Brujas.

Ballesteros, B., Meloni, F., \& Baccheta, G. (2015). Manual para la propagación de pántulas autóctonas mediterráneas seleccionadas. Ecoplantmed, ENPI, CBC-MED.

Conchago, J. (2008). Inventario Forestal de Especies Nativas del Bosque Protector de Río Blanco parroquía Quimiag, cantón Riobamba. Riobamba: Honorable Consejo Provincial de Chimborazo.

Edmon. (1997). Suelo y Medio Ambiente en Invernaderos. Sevilla, España: Consejería de Agricultura y Pesca.

Erazo, Norma (2009). Entrevista personal (Laboratorio Biológico- ESPOCH).

Estación Meteorológica de la ESPOCH (2016). Datos climáticos de la serie 1984-2014.Riobamba.

Fernández B. J.M. y M.R. Quezada 1992. Producción de planta con uso de materiales plásticos, $3^{\circ}$ curso nacional de plásticos en la agricultura, C.I.Q.A. Saltillo Coahuila

Guevara, M., \& Villacrés, M. (1999). Producción de Plántulas en Viveros. Quito: Fundación Forestal Juan Manuel Durini. Nota Técnica №7.

Jará, L., \& Ordóñez, G. (1999). Curso de Manejo de Semillas y Viveros Forestales. Santo Domingo de los Colorados: PROFAFOR.

Laboratorio de suelos de la Facultad de Recursos Naturales de la ESPOCH. (2014). Resultados de Análisis de sustratos del ensayo. Provincia de Chimborazo. Riobamba.

Luteyn, J. L. (1999). Páramos, a checklist of plant diversity, geografhical distribution, and botanical literature. Mem.New York Bot.Gard. 
Ministerio del Ambiente. (2011). Normativa forestal Bosque Andino. Obtenido de http://www.ambiente.gob.ec/sites/default/files/archivos/normativaforestal/bosquesandinos.p df

Ministerio del Ambiente del Ecuador. (2012). Sistema de clasificación de los ecosistemas del Ecuador continental. Quito: Subsecretaria de Patrimoni Natural.

MAE. (2014). Plan Nacional de Reforestación. Documento. Quito, Ecuador.

Mulawarman, J. M., Roshetko, S. M., Sasongko, \& D, I. (2003). Tree seed management- seed sources, seed collection and seed handling: a field manual for field workers and farmers. Bogor, Indonesia: International Centre for Research in Agroforetry (ICRAF) and Winrock International.

Niembro, R. A., \& Fierros, A. M. (1990). Factores ambientales que controlan la germinación de las semillas de pinos. Chapingo: En: Memoria. Mejoramiento Genético y Plantaciones Forestales. Centro de Genética Forestal, A.C.

Prado, L., \& Valdebenito, H. (2000). Contribución a la fenología de especies forestales nativas andinas de Bolivia y Ecuador. Quito; Ecuador: Intercooperation.

Ruiz, W. (2014). Evaluación de Tres Sustratos Para la Propagación de Tilo (Tilia cordata Mill) y Sacha Capulí (Vallea stipularis) con la Aplicación de Tres Hormonas de Enraizamiento en el Vivero del GAD Municipal del Cantón Mejía 2014 (Trabajo de pregrado). Latacunga: Universidad Técnica de Cotopaxi.

Sklenář P, J. L., Luteyn, C., Ulloa Ulloa, P. M., Jørgensen, \& M.O, D. (2005). Flora genérica de los páramos-Guía ilustrada de las plantas vasculares. Mem New York Bot. Gard.

Trujillo, E. (2010). Manual de Árboles. Investigaciones Forestales. Bogotá, Colombia.

Vademécum Agrícola. (2008). Quito-Ecuador: Edifarm.

Vázquez, C., \& Batis, A. I. (1996). La restauración de la vegetación, árboles exóticos vs árboles nativos. Ciencias, (043).

Vega, J. A. (1986). Secuestro de carbono en plantaciones de eucal Estudio de algunos factores que influyen en la producción de Pinus montezumae Lamb. En vivero (Tesis de maestría). Chapingo, México. 